\title{
Unilateral versus bilateral adnexal disease in stage III and stage IV endometriosis does not affect pregnancy outcome after operative laparoscopy
}

\author{
Mostafa Abuzeid • Abeer Ahmed • Khaled Sakhel • \\ Riham Alwan • Mohammad Ashraf • \\ Mohammed Mitwally • Michael Diamond
}

Received: 14 April 2008 /Accepted: 27 June 2008/Published online: 23 August 2008

(C) Springer-Verlag 2008

\begin{abstract}
To study the effect of unilateral versus bilateral adnexal involvement on the pregnancy rate after operative laparoscopy. Historic cohort study. A total of 143 patients with advanced stages of endometriosis (stage III and IV) who underwent operative laparoscopy for infertility treatment were categorized into those with unilateral versus bilateral adnexal disease. Forty-three patients had primarily unilateral (group 1) and 100 had bilateral disease (group 2). Follow-up was up to 2 years. There was no significant difference between group 1 and group 2 with respect to
\end{abstract}

Support: None

M. Abuzeid $(\bowtie) \cdot$ M. Ashraf

Division of Reproductive Endocrinology and Infertility, Department of OB/GYN,

Hurley Medical Center,

Flint, MI, USA

e-mail: reprod1@hurleymc.com

M. Abuzeid $\cdot$ M. Ashraf

College of Human Medicine,

Michigan State University,

E Lansing, MI, USA

A. Ahmed

Department of $\mathrm{OB} / \mathrm{GYN}$,

Hurley Medical Center,

Flint, MI, USA

M. Abuzeid $\cdot$ K. Sakhel $\cdot$ R. Alwan · M. Ashraf

IVF Michigan PC,

Rochester Hills, MI, USA

M. Mitwally $\cdot$ M. Diamond

Division of Reproductive Endocrinology and Infertility,

Wayne State University,

Detriot, MI, USA pregnancy rates $(28 \%$ vs $31 \%)$, delivery rates $(21 \%$ vs $23 \%)$, miscarriage rates ( $25 \%$ vs $13 \%)$, and ectopic rates $(0 \%$ vs $13 \%)$. Cumulative conception rates after up to 24 cycles of natural intercourse and up to three IUI cycles were $59 \%$ and $49 \%$ for group 1 and group 2, respectively. Operative laparoscopy for advanced stages of endometriosis appears to be an effective treatment modality for establishing a pregnancy in infertile patients. Pregnancy and delivery rates are not different in patients with unilateral or bilateral adnexal involvement.

Keywords Advanced endometriosis · Pregnancy outcome · Laparoscopic surgery $\cdot$ Unilateral $\cdot$ Bilateral

\section{Introduction}

Although stage III and IV endometriosis suggest advanced disease, endometrial implants and adhesions are frequently not symmetrically distributed in the pelvis [1] allowing for the possibility that one adnexa may be relatively free of mechanical factors of infertility. The fact that one adnexa may have less disease may positively influence the pregnancy rate reported as a result of surgical treatment of advanced endometriosis. That is to say, the pregnancy occurs as a result of ovulation/tubal ovum pickup from the side with less adnexal pathology. However, in such cases the resulting pregnancy may be due to the effect of ablation of endometrial implant, as in patients with minimal or mild endometriosis [2]. If such a hypothesis is true, the pregnancy rate should be much less in patients with bilateral adnexal involvement. To examine this hypothesis, we therefore studied the effect of unilateral versus bilateral adnexal involvement on the pregnancy rate after operative laparos- 
copy for infertile patients with advanced endometriosis. We also studied the pregnancy and delivery rates in these two groups and in the total population after different infertility treatment methods were used.

\section{Materials and methods}

This is a retrospective historical cohort study conducted after obtaining approval by the local institutional research board of Wayne State University. The study was conducted at a private infertility center (IVF Michigan). All patients with advanced stages of endometriosis (stage III and IV) who underwent operative laparoscopy for infertility treatment between April 1993 and December 2003 were studied. Endometriosis was staged at the time of surgery according to the revised American Fertility Society classification (rAFS) [3]. Patients whose husbands had severe male factor (count less than 10 million/ml, and/or motility less than $20 \%$, and/or very poor morphology) and patients with cycle day $3 \mathrm{FSH}$ level higher than $12 \mathrm{mIU} / \mathrm{ml}$ were excluded. Patients with past history of pelvic inflammatory disease (PID), history of sexually transmitted disease and/or positive Chlamydia trachomatis serology (IgG/IgM) were excluded. A total of 143 consecutive patients who satisfied the criteria were included in our study. Patients were characterized into those with unilateral versus bilateral adnexal disease. Adnexal disease was defined as mechanical distortion of the adnexa due to the presence of deepseated endometrioma(s) and/or adhesions of the ovary to the ovarian fossa and/or extensive peritubal and/or periovarian adhesions. Unilateral disease was defined as similar anatomical distortions mechanically affecting only one adnexa. Superficial implants of the opposite adnexa, in the absence of adhesions or endometrioma(s), did not disqualify a patient as having primarily unilateral disease. Patients designated as being in the bilateral group had significant ovarian and/or tubal pathology distorting both adnexae. Using these criteria, 43 patients had primarily unilateral and 100 had bilateral disease.

Surgery was performed by the same surgeon (M. I. A.) under similar operative setting in all patients. The same surgical techniques and procedures were applied in all patients. When suspicious spots of endometriosis were found anywhere in the pelvis, in the majority of cases, argon beam coagulator (Birtcher Medical System, Irvine, CA, USA) was used to ablate the spots seen. If endometriosis was found on the pelvic sidewall near the ureter, on the bladder or bowels, $\mathrm{CO}_{2}$ laser (Sharplan Lasers, Inc., Allendale, NJ, USA) vaporization was performed. Salpingo-ovariolysis was performed using blunt dissection of the ovary from the pelvic sidewall, and scissors and/or monopolar diathermy needle tip to excise scar tissue from the tubes and ovaries. If an endometrioma of more than $1 \mathrm{~cm}$ was found, excision of the cyst wall was done and reconstruction of the ovarian cortex was performed with one or two interrupted sutures using 3-0 Vicryl. If an endometrioma of less than $1 \mathrm{~cm}$ was found, it was opened, evacuated, and the lining was ablated with the argon beam coagulator. If fimbrial phimosis or hydrosalpinges were found, fimbrioplasty or salpingostomy was performed, respectively. Starting in 1999, temporary ovarian suspension was performed when laparoscopic surgery was performed for advanced endometriosis in an attempt to reduce the risk of recurrence of adhesions between ovarian fossa and ovaries [4].

Following surgery, Clomid was given to patients with anovulatory disorder. Intrauterine insemination (IUI) after controlled ovarian hyperstimulation $(\mathrm{COH})$ was performed if male factor or ovulatory disorder resistant to Clomid (not responding by ovulation) was present, or after 6 months of natural intercourse. Some patients opted to start infertility intervention in the form of $\mathrm{COH}+\mathrm{IUI}$ or in vitro fertilization and embryo transfer (IVF-ET) directly after surgery.

During IUI, ovarian hyperstimulation was achieved using mid-luteal gonadotropin-releasing hormone agonist (GnRH-a) pituitary down regulation followed by gonadotropin injections starting on the second or third day of the menstrual cycle. Ten thousand units of human chorionic gonadotropin (hCG) was given when the dominant follicle reached a diameter of $17 \mathrm{~mm}$, and IUI was performed $42 \mathrm{~h}$ later. For IVF/ET, controlled ovarian hyperstimulation was applied according to a long GnRH-a pituitary down regulation protocol. In the first few years of this series, traditional IVF was performed. However, intracytoplasmic sperm injection (ICSI) was performed in all patients in the last 5 years of this series.

In all patients studied, we calculated endometriosis scores in five categories: cul-de-sac, peritoneum, ovaries, tubal, and grand total. After excluding patients who conceived with IVF-ET (in whom pregnancy is not secondary to surgery), we compared these scores in patients who conceived and those who failed to conceive in each group and in the total population studied. In addition, after excluding 31 patients who conceived after IVF/ET, the data were then analyzed with respect to the effect of the underlying etiology.

Pregnancy was confirmed by vaginal ultrasonography and delivery verified by phone follow-up. Patients were followed until delivery if pregnant, or for a period of 24 months following surgery if not pregnant. Data were expressed as means $\pm \mathrm{SD}$. Student's $t$-test, chi square analysis, and Kruskal-Wallis one way anova analysis were used for statistical analysis where appropriate. Cumulative conception rate (CCR) was calculated using life table analysis. To determine if there was any statistical signifi- 
cance in CCR between the two groups, further survival analysis was performed; the Kaplan-Meier survival analysis log rank test was used.

\section{Results}

The mean age at laparoscopy was $31.8 \pm 4.5$ years. The mean duration of infertility was $3.4 \pm 2.7$ years. Table 1 shows the demographic data of both groups. The two groups did not differ significantly in age and duration of infertility, although the latter approached significance with a tendency for longer duration of infertility in the unilateral group. A significantly higher number of patients had primary infertility in the unilateral group. In addition, there were no significant differences in the underlying etiologies of infertility between the two groups. Of the patients studied, 78 patients $(55 \%)$ had primary infertility. Fortythree patients $(30.1 \%)$ had endometriosis as their sole cause of infertility and 100 patients $(69.9 \%)$ had other infertility factors (e.g., mild male factor or ovulatory disorder) in addition to endometriosis.

Tables 2, 3 and 4 illustrate pregnancy outcome after spontaneous conception, conception after IUI $+\mathrm{COH}$, and combination of the two methods, respectively. There was no significant difference in the pregnancy rates between the unilateral versus bilateral groups after spontaneous $(19 \%$ vs $21 \%$ ), IUI $+\mathrm{COH}$ ( $40 \%$ vs $53 \%$ ), and combined methods ( $28 \%$ vs $31 \%$ ), respectively. In addition, there was no significant difference in delivery rates between the two groups after spontaneous (14\% vs $15 \%)$, IUI $+\mathrm{COH}(30 \%$ vs $42 \%)$, or combined ( $21 \%$ and $23 \%$ ) conceptions, respectively. Furthermore, there was no significant difference in miscarriage rates and ectopic pregnancy rates between the two groups, according to the method used to achieve conception. The overall conception rate, delivery rate, miscarriage rate, and ectopic pregnancy rate were $20 \%, 15 \%, 17 \%$, and $10 \%$ with spontaneous conception; $42 \%, 38 \%, 14 \%$, and $7 \%$ after $\mathrm{COH}+\mathrm{IUI}$; and $30 \%, 22 \%$, $16 \%$ and $9 \%$ after combined methods, respectively. There were no significant differences in the CCR between unilateral versus bilateral groups after spontaneous conception $(53 \%$ vs $43 \%)$, IUI $+\mathrm{COH}$ ( $47 \%$ vs $81 \%$ ), or combined (59\% vs $49 \%)$, respectively.

Some of the patients elected to proceed with IVF/ET shortly after surgery when they were told of the extent of their endometriosis. Others tried spontaneously and/or IUI+ $\mathrm{COH}$ for a few cycles before they decided to proceed with IVF/ET. Fifty-one patients underwent 75 cycles of IVF/ET during the study period. There was no significant difference between the two groups in the pregnancy rate per patient ( $67 \%$ vs $58 \%$ ), the delivery rate per patient ( $56 \%$ vs $49 \%$ ), miscarriage rate $(8 \%$ vs $11 \%)$, and ectopic pregnancy rate ( $8 \%$ vs $5 \%)$, respectively.

Of the 51 patients who underwent IVF/ET, 31 patients conceived. The pregnancy and delivery rates per patient were $61 \%$ and $51 \%$, respectively, while the miscarriage and ectopic pregnancy rates were $10 \%$ and $7 \%$, respectively.

After excluding 31 patients who conceived after IVF-ET, the data were then analyzed and the pregnancy outcome after combined methods (spontaneous conception and IUI+ $\mathrm{COH})$ were calculated. The pregnancy rate and delivery rate were $39 \%$ and $29 \%$ in the unilateral group, $38 \%$ and $28 \%$ in the bilateral group and $38 \%$ and $29 \%$ in the total population, respectively. We also compared the pregnancy rates between the two groups according to the underlying etiology. There was no significant difference in the pregnancy rate in relation to the underlying etiology between the two groups. Above $50 \%$ of patients achieved pregnancy and approximately $40 \%$ delivered in both groups when all possible infertility interventions were used. When all possible infertility treatment methods were used, cumulative conception rate and cumulative delivery rate after 24 months were $66 \%$ and $62 \%$ for group 1, 69\% and $68 \%$ for group 2 , and $76 \%$ and $67 \%$ for the total population studied, respectively.

We compared endometriosis scores in patients who conceived and those who failed to conceive in the two groups and in the total population after excluding patients who conceived with IVF-ET. There was no significant difference in the endometriosis score in the unilateral group, while in both the bilateral group and in the total

Table 1 Demographic data

\begin{tabular}{|c|c|c|c|c|}
\hline & Unilateral & Bilateral & $P$ value & Total \\
\hline Number of patients & 43 & 100 & & 143 \\
\hline Age & $32.0 \pm 4.7$ & $31.8 \pm 4.5$ & NS & $31.8 \pm 4.5$ \\
\hline Duration of infertility & $4.0 \pm 3.0$ & $3.1 \pm 2.5$ & 0.052 & $3.4 \pm 2.7$ \\
\hline \multicolumn{5}{|l|}{ Type of infertility } \\
\hline Primary infertility & $31(72 \%)$ & $47(47 \%)$ & $<0.01$ & $78(54.5 \%)$ \\
\hline \multicolumn{5}{|l|}{ Etiology of infertility } \\
\hline Endometriosis alone & $8(18.6 \%)$ & $35(35 \%)$ & NS & $43(30.1 \%)$ \\
\hline Endometriosis plus other infertility factors & $35(81.4 \%)$ & $65(65 \%)$ & NS & $100(69.9 \%)$ \\
\hline
\end{tabular}


Table 2 Pregnancy outcome after spontaneous conception

\begin{tabular}{|c|c|c|c|c|c|c|}
\hline & Number of patients & Pregnant (\%) & Delivered (\%) & Miscarried (\%) & Ectopic (\%) & $\mathrm{CCR}^{\mathrm{a}}$ \\
\hline Unilateral & 43 & $8(19)$ & $6(14)$ & $2(25)$ & $0(0)$ & $53 \%$ \\
\hline Bilateral & 100 & $21(21)$ & $15(15)$ & $3(14)$ & $3(14)$ & $43 \%$ \\
\hline Total & 143 & $29(20)$ & $21(15)$ & $5(17)$ & $3(10)$ & $46 \%$ \\
\hline
\end{tabular}

${ }^{\text {a }}$ Cumulative conception rate up to 24 months after surgery

population there was significantly lower ovarian scores $(P=$ 0.007 and $P=0.001$, respectively) and grand total scores ( $P=$ 0.001 and $P=0.001$, respectively) in the conceived versus non-conceived. No difference was detected in the tubal scores among the unilateral group, bilateral group, and the total population.

\section{Discussion}

Although endometriosis is present in $25-50 \%$ of infertile women, [5, 6] the majority of these patients have early stages of endometriosis. Since mechanical factors interfering with ovum pick up play an important role in the mechanisms of infertility in patients with advanced stages of endometriosis, the majority of these patients require either surgical correction or IVF/ET to achieve pregnancy. Many studies reported very low cumulative conception rate (CCR) in patients with advanced stages of endometriosis without surgical treatment [7-10] compared to CCR in patients with early stages of endometriosis [11-13]. Olive and colleagues reported no pregnancies among women with severe endometriosis with expectant management, as compared to $52 \%$ with mild endometriosis and expectant management during a 25-month follow-up [9]. In addition, several reports suggested good CCR after surgical treatment of advanced endometriosis both by laparotomy and laparoscopy [14-16]. Other reports suggested no difference in the pregnancy outcome whether laparotomy or laparoscopy was utilized. [14, 17]

Now, for more than a decade, laparoscopy has become the gold standard of conservative surgical treatment of infertility patients with endometriosis [14-16]. It allows the surgeon to reach a diagnosis and allows him/her to proceed with treatment of endometriosis and/or adhesions at the time of surgery. Improvement in fecundity rate and CCR have been reported after laparoscopic surgical treatment for infertile patients with both early [2] and advanced stages of endometriosis [14, -18]. However, none of these studies evaluated the effect of unilateral versus bilateral adnexal involvement on the chances of achieving pregnancy postoperatively.

Our data indicate that surgical treatment for advanced endometriosis is effective even when bilateral adnexal involvement is present. Our results, with a CCR of $53 \%$ after spontaneous cycles, are similar to the results reported by other investigators, which suggested that approximately $50 \%$ of patients conceived after laparoscopic surgery for advanced endometriosis [19]. In addition, our results are in agreement with others that laparoscopic surgery can improve pregnancy rates in patients with advanced disease $[18,20]$. In a recent report by Littman et al., of the 19 patients with advanced stage of endometriosis, eight conceived (seven spontaneously and one after Clomid/ IUI) [42\%] [21]. In our study, when both spontaneous and IUI $+\mathrm{COH}$ methods of conception were used, we were able to achieve a CCR of $59 \%, 49 \%$, and $52 \%$ for the unilateral group, bilateral group, and the total population studied, respectively. However, our data also suggest that when ovarian endometriosis scoring is high in bilateral disease, the chances are less favorable. It may partly explain the difference in pregnancy rate after laparoscopic surgical corrections of advanced endometriosis in various reports. Busacca et al. reported a cumulative conception rate of $51 \%$ and $17 \%$ after operative laparoscopy for stage III and IV endometriosis, respectively [22]. Similar to our data, this report suggests the more advanced (higher scores), the less likely the chances of pregnancy. Similarly, Adamson et al.

Table 3 Pregnancy outcome after IUI+COH

\begin{tabular}{|c|c|c|c|c|c|c|c|c|c|}
\hline & $\begin{array}{l}\text { Number } \\
\text { of patients }\end{array}$ & $\begin{array}{l}\text { Number } \\
\text { of cycles }\end{array}$ & $\begin{array}{l}\text { Number } \\
\text { pregnant } \\
\text { per cycle }(\%)\end{array}$ & $\begin{array}{l}\text { Number } \\
\text { pregnant } \\
\text { per patient }(\%)\end{array}$ & $\begin{array}{l}\text { Number } \\
\text { delivered } \\
\text { per cycle }(\%)\end{array}$ & $\begin{array}{l}\text { Number } \\
\text { delivered } \\
\text { per patient (\%) }\end{array}$ & $\begin{array}{l}\text { Number } \\
\text { miscarried } \\
(\%)\end{array}$ & $\begin{array}{l}\text { Number } \\
\text { ectopic (\%) }\end{array}$ & $\mathrm{CCR}^{\mathrm{a}}$ \\
\hline Unilateral & 10 & 19 & $4(21)$ & $4(40)$ & $3(16)$ & $3(30)$ & $1(25)$ & $0(0)$ & $47 \%$ \\
\hline Bilateral & 19 & 32 & $10(31)$ & $10(53)$ & $8(25)$ & $8(42)$ & $1(10)$ & $1(10)$ & $81 \%$ \\
\hline Total & 29 & 51 & $14(28)$ & $14(42)$ & $11(22)$ & $11(38)$ & $2(14)$ & $1(7)$ & $73 \%$ \\
\hline
\end{tabular}

${ }^{\text {a }}$ Cumulative conception rate up to 24 months after surgery 
Table 4 Pregnancy outcome after combined methods (spontaneous conception and IUI $+\mathrm{COH}$ )

\begin{tabular}{lccccc}
\hline & $\begin{array}{l}\text { Number } \\
\text { of patients }\end{array}$ & $\begin{array}{l}\text { Number } \\
\text { pregnant } \\
\text { per patient (\%) }\end{array}$ & $\begin{array}{l}\text { Number } \\
\text { delivered } \\
\text { per patient (\%) }\end{array}$ & $\begin{array}{l}\text { Number } \\
\text { miscarried (\%) }\end{array}$ & $\begin{array}{l}\text { Number } \\
\text { ectopic (\%) }\end{array}$ \\
\hline Unilateral & 43 & $12(28)$ & $9(21)$ & $3(25)$ & $0(0)$ \\
Bilateral & 100 & $31(31)$ & $23(23)$ & $4(13)$ & $4(13)$ \\
Total & 143 & $43(30)$ & $32(22)$ & $7(16)$ & $49 \%$ \\
\hline
\end{tabular}

${ }^{\text {a }}$ Cumulative conception rate up to 24 months after surgery

reported that extensive endometriosis with higher ASRM score $(>71)$, was associated with reduced pregnancy rates [23]. Other reports suggested that older patients and those with stage IV did not conceive as often [19].

In one recent study, Maruyama [24] found that cumulative rates of pregnancy after laparoscopic treatment of endometriosis are about 35\%. Maruyama found a significantly lower cumulative pregnancy rate in a group of patients with bilateral tubal adhesions compared with those patients with unilateral or no tubal adhesions. However, he did not find a difference in the pregnancy outcomes of those patients with minimal/mild endometriosis versus moderate/severe endometriosis if the endometriosis did not include tubal disease. This suggests that endometrial implants resulting in mechanical factors interfering with tubal function may have a stronger impact on pregnancy outcome than the extent of endometrial disease as reflected merely in staging. This may explain the relative success of laparoscopic correction of mechanical factors in even those patients with severe stages of endometriosis. In our study, when we evaluated the effect of tubal involvement, we could not find any significant difference between patients who conceived and those who failed to conceive. Such findings were maintained in both unilateral and bilateral groups and also in the total population studied. We attribute such findings to the fact that we excluded from this study all patients who had history of PID and/or those who had positive serology for Chlamydia trachomatis. Since endometriosis is a disease of the peritoneum, surgical correction of any tubal pathology in our patients may have a better prognosis than in patients with PID.

Our data also suggest that $\mathrm{IUI}+\mathrm{COH}$ can enhance pregnancy chances after laparoscopic surgery for advanced endometriosis. In our study, approximately one-third of patients with advanced endometriosis were able to conceive within 2 years after operative laparoscopy as a result of spontaneous trials and/or $\mathrm{IUI}+\mathrm{COH}$. Other reports suggested similar results when $\mathrm{IUI}+\mathrm{COH}$ was used after operative laparoscopy for early and advanced endometriosis [25-27]. Therefore, it could be offered for two to three cycles either immediately following surgery or after three to four failed cycles of spontaneous trials.
Our data suggest consistent excellent results of IVF-ET after operative laparoscopy. Therefore, our result is in favor of the IVF-ET procedure after surgery for endometriosis. IVF-ET should be considered after operative laparoscopy whenever there is marked bilateral ovarian involvement and/or failure of conception spontaneously, or after a few trials of $\mathrm{IUI}+\mathrm{COH}$. In addition, it should be considered from the outset in patients with additional infertility factors; e.g., male factor, advanced female age, and prolonged duration of infertility. Recently, Littman et al. reported eight IVF pregnancies (five in patients with stage III or IV) in patients with endometriosis after operative laparoscopy who had two failed IVF cycles on average. However, the literature is not conclusive on this issue [28-30]. It is worth of note that ICSI is being utilized more frequently in these patients in view of reports of decreased fertilization rate after traditional IVF/ET [29, 31, 32]. Some reports suggested that ICSI can overcome apparent defects in oocytes derived from endometriosis patients [33].

However, our study has some limitations including its retrospective nature, the heterogenous group of patients studied with the fact that only $30 \%$ of the patients had endometriosis as their only cause of infertility and the fact that, following surgery, the patients were not carried forward in a similar manner. On the other hand, this study was carried out by one surgeon at one unit and therefore, represents consistent management over the period of the study. In addition, it is extremely difficult to answer the question posed in this study by limiting the data to only those with the single diagnosis of endometriosis. Furthermore, the fact that spontaneous pregnancy rate is similar in both groups is very suggestive that laparoscopic surgical treatment is as effective in the presence of bilateral adnexal involvement as in unilateral adnexal disease irrespective of the presence of additional infertility factors.

In conclusion, the data in our study suggest that conservative laparoscopic surgery for advanced stages of endometriosis appears to be an effective treatment modality in patients with both unilateral and bilateral adnexal involvement. However, a large prospective study is needed to confirm our findings. In addition, IUI+COH may enhance chances of conception in patients with advanced endometri- 
osis after operative laparoscopy. However, as expected, the more advanced the disease, the lesser the likelihood of pregnancy after surgical correction. Such patients should be counseled to consider other opportunities including proceeding with IVF-ET, which can achieve excellent results.

Acknowledgments The abstract of this paper was presented in part at the Endometriosis 2000 7th Biennial World Congress, London, May 14-17, 2000

Abstract presented in part at the ASRM/CFAS meeting, Montreal Canada October 15-19, Fertil Steril (84): Suppl 1, S198, Sept. 2005

The authors would like to thank Jenny LaChance for her assistance in statistical analysis and Cheryl Anderson for her assistance in the preparation of this manuscript.

\section{References}

1. Chapron C, Chopin N, Borghese B, Foulot H, Dousset B, VacherLavenu MC, Vieira M, Hasan W, Bricou A (2006) Deeply infiltrating endometriosis: pathogenetic implications of the anatomical distribution. Hum Reprod 21(7):1839-1845

2. Marcoux S, Maheux R, Beruse S, The Canadian Collaborative Group on Endometriosis (1997) Laparoscopic surgery in infertile women with minimal or mild endometriosis. New Eng J Med 337 (4):217-222

3. American Society for Reproductive Medicine (1997) Revised American Society for Reproductive Medicine classification of endometriosis: 1996. Fertil Steril 67:817-821

4. Abuzeid MI, Ashraf M, Shamma FN (2002) Temporary ovarian suspension at laparoscopy for prevention of adhesions. J Am Assoc Gynecol Laparosc 9(1):98-102

5. Strathy JH, Molgaard CA, Coulam CB, Melton LJ (1982) Endometriosis and infertility: a laparoscopic study of endometriosis among fertile and infertile women. Fertil Steril 38:676-672

6. Nezhat C, Crowgey SR, Garrison CP (1986) Surgical treatment of endometriosis via laser laparoscopy. Fertil Steril 45:778-783

7. Garcia C, David SS (1977) Pelvic endometriosis: infertility and pelvic pain. Am J Obstet Gynecol 129:740-747

8. Hull MGR (1990) Indications for assisted conception. Br Med Bull 46:580-595

9. Olive DL, Stohs GF, Metzger DA, Franklin RR (1985) Expectant management and hydrotubations in the treatment of endometriosisassociated infertility. Fertil Steril 44:35-41

10. Olive DL, Lee KL (1986) Analysis of sequential treatment protocols for endometriosis-associated infertility. Am J Obstet Gynecol 154:613-619

11. Badawy SZA, Elbakry MM, Samuel F, Dizer M (1988) Cumulative pregnancy rates in infertile women with endometriosis. J Reprod Med 33:757-760

12. Hull MEO, Moghissi KS, Magyar DF, Hayes MF (1987) Comparison of different treatment modalities of endometriosis in infertile women. Fertil Steril 47:40-44

13. Portuondo JA, Echanojauregui AD, Herran C, Alijarte I (1983) Early conception in patients with untreated mild endometriosis. Fertil Steril 39:22-24

14. Adamson GD, Subak LL, Pasta DJ et al (1992) Comparison of $\mathrm{CO}_{2}$ laser laparoscopy with laparotomy for treatment of endometriomata. Fertil Steril 57:965-973

15. Adamson GD, Hurd SJ, Pasta DJ et al (1993) Laparoscopic endometriosis treatment: is it better? Fertil Steril 59:35-44
16. Dlugi AM, Saleh WA, Jacobsen G (1992) KTP/532 laser laparoscopy in the treatment of endometriosis-associated infertility. Fertil Steril 57(6):1186-1193

17. Al-Inany HG, Crosignani PG, Vercellini P (2000) Evidence may change with more trials: concepts to be kept in mind [Letters]. Hum Reprod 15:2447-2448

18. Jacobson TZ, Barlow DH, Koninckx PR, Olive D, Farquhar C (2002) Laparoscopic surgery for subfertility associated with endometriosis. Cochrane Database Syst Rev:CD001398

19. Adamson GD, Pasta DJ (1994) Surgical treatment of endometriosisassociated infertility: meta-analysis compared with survival analysis. Am J Obstet Gynecol 171:1488-505

20. Mohr C, Nezhat FR, Nezhat CH, Siedman DS, Nezhat C (2005) Fertility considerations in patients with bowel endometriosis. J Laparoendosc Surg 9:16-24

21. Littman E, Guidice L, Lathi R, Berker B, Milki A, Nezhat C (2005) Role of laparoscopic treatment of endometriosis in patients who have failed in vitro fertilization cycles. Fertil Steril 84:1574-1578

22. Busacca M, Bianchi S, Agnoli B, Candiani M, Calia C, De Marinis S, Vignali M (1999) Follow-up of laparoscopic treatment of stage III-IV endometriosis. J Am Assoc Gynecol Laparosc 6 (1):55-58

23. Adamson GD, Pasta DJ (2002) Pregnancy rates can be predicted by validated endometriosis fertility index (EFI). Fertil Steril 77 (Suppl 1):S48

24. Maruyama M, Osuga Y, Momoeda M, Yano T, Tsutsumi O, Taketani Y (2000) Pregnancy rates after laparoscopic treatment. Differences related to tubal status and presence of endometriosis. $\mathrm{J}$ Reprod Med 45(2):89-93

25. Deaton JL, Gibson M, Blackmer KM et al (1990) A randomized, controlled trial of clomiphene citrate and intrauterine insemination in couples with unexplained infertility or surgically corrected endometriosis. Fertil Steril 54:1083-1088

26. Fedele L, Bianchi S, Marchini M et al (1997) Superovulation with human menopausal gonadotropins in the treatment of infertility associated with minimal or mild endometriosis: a controlled randomized study. Fertil Steril 68:8-12

27. Lodhi S, Fattah A, Abozaid T, Murphy J, Formantini E, Sasy M, Barber K, Abuzeid M (2004) Gamete intra-fallopian transfer or intrauterine insemination after controlled ovarian hyperstimulation for treatment of infertility due to endometriosis. Gynecol Endocrinol 19:152-159

28. Barnhard K, Dunsmoor-Su R, Coutifaris C (2002) Effect of endometriosis on in vitro fertilization. Fertil Steril 77:1148-1155

29. Guzick DS, Silliman NP, Adamson GD, Buttram VC, Canis M, Malinak LR, Schenken RS (1997) Prediction of pregnancy in infertile women based on the American Society for Reproductive Medicine's revised classification of endometriosis. Fertil Steril 67:822-829

30. Aboulghar MA, Mansour RT, Serour GI, Al-Inany HG, Aboulghar MM (2003) The outcome of in vitro fertilization in advanced endometriosis with previous surgery: a case-controlled study. Am J Obstet Gynecol 188:371-375

31. Wardle PG, Foster PA, Mitchell JD, McLaughlin EA, Sykes JA, Corrigan E, Hull MG, Ray BD, McDermott A (1986) Endometriosis and IVF: effect of prior therapy (letter). Lancet 1 (8475):276-277

32. Dmowski PW, Gleicher N (1998) Does endometriosis have an adverse effect on fertilization in vitro and the IVF outcome? J Assist Reprod Genet 15(9):527-529

33. Bukulmez O, Yarali H, Gurgan T (2001) The presence and extent of endometriosis do not effect clinical pregnancy and implantation rates in patients undergoing intracytoplasmic sperm injection. Euro J Obstet Gynec Reprod Biol 96:102-107 